\title{
Fluctuation of the Endogenous IAA Level in Rice during Its Life Cycle
}

\author{
Masatomo Kobayashi, Akira SaKurai, Hitoshi SaKa* \\ and Nobutaka TAKAHASHI** \\ The Institute of Physical and Chemical Research, Wako-shi, \\ Saitama 351-01, Japan \\ * National Institute of Agrobiological Resources, Tsukuba-shi, \\ Ibaraki 305, Japan \\ ** Department of Agricultural Chemistry, The University of Tokyo, \\ Bunkyo-ku, Tokyo 113, Japan \\ Received November 16, 1988
}

\begin{abstract}
Endogenous auxins in the shoots and ears of rice were investigated. Indole-3-acetic acid (IAA) and indole-3-carboxylic acid (ICA) was identified by GC-MS, the endogenous level of IAA being much higher than that of ICA. To analyze the fluctuation of endogenous IAA level throughout the life cycle of rice, a rapid and effective procedure, using HPLC and a fluorescence detector, was developed. The level of IAA in shoots was $10 \sim 26 \mathrm{ng} / \mathrm{g} \mathrm{fr}$. wt., while that in ears was 10 to 100 times higher. The level of IAA conjugate in ears was also much higher than that in shoots. These results show that the biosynthesis of IAA occurs at an early stage of seed development, and it is also suggested that IAA may play a role in regulating the reproductive growth of rice.
\end{abstract}

In a series of investigations on the endogenous plant hormones of rice (Oryza sativa L.), the occurrence and seasonal changes in the levels of gibberellins, cytokinins and abscisic acid have been clarified. ${ }^{1 \sim 5)}$ The analysis of gibberellins revealed that the level of gibberellin $\mathrm{A}_{4}$ in anthers at the anthesis stage was extremely high. ${ }^{4)}$ This result showed that gibberellin may play an important role in regulating the physiological phenomena at the heading and/or anthesis stage. Endogenous levels of cytokinins and abscisic acid in ears were also much higher than those in vegetative tissue, ${ }^{2,5}$ suggesting that these plant hormones may regulate the development of seeds.

Bandurski et al. ${ }^{6)}$ analyzed endogenous IAA in mature seeds of rice and showed that the contents of IAA and its conjugate were 1703 and $2739 \mu \mathrm{g} / \mathrm{kg}$, respectively. However, a comparison of the endogenous auxin levels between vegetative and reproductive tissues of rice had not been made. Thus, we analyzed the endogenous auxins in rice to reveal the roles of auxins in regulating the growth of rice. In this paper, we report the identification of endogenous auxins, and the fluctuation of the endogenous levels of IAA and its conjugate during the life cycle of rice.

\section{Materials and Methods}

Plant materials. Oryza sativa L. cultivar Nihonbare (japonica variety) was grown in a paddy field of the National Institute of Agrobiological Resources in 1985 and 1986. Whole plants were collected at the 3rd-leaf stage, 6th-leaf stage, maximum tillering stage and anthesis stage in each year, and at the panicle initiation stage in 1986. The roots were removed from each sample except those collected at the 3rd-leaf stage. The ears were collected at the beginning of the anthesis stage, one week after anthesis (beginning of the milky ripening stage) and fourty to fifty days after anthesis (end of the ripening stage). Table I summarizes the features of the collected samples, which were immersed in methanol and stored at $-20^{\circ} \mathrm{C}$

Abbreviations: BHT, 2,6-di-tert-butyl-p-crezol; GC-MS, combined gas chromatography-mass spectrometry; HPLC, high-performance liquid chromatography; IAA, indole-3-acetic acid; ICA, indole-3-carboxylic acid; IPA, indole3-propionic acid; IBA, indole-3-butyric acid; TMSi, trimethylsilyl. 
Table I. Plant Materials for iaA Analysis

\begin{tabular}{lclcc}
\hline Stage & $\begin{array}{c}\text { Days after } \\
\text { sowing }\end{array}$ & $\begin{array}{c}\text { Collected } \\
\text { organ }\end{array}$ & $\begin{array}{c}\text { Number of } \\
\text { organs }\end{array}$ & $\begin{array}{c}\text { Weight of } \\
\text { organs (g) }\end{array}$ \\
\hline in 1985 & & & & \\
$\quad$ 3rd-leaf & 17 & Whole plant & 609 plants & 122 \\
6th-leaf & 32 & Shoot & 275 plants & 61 \\
$\quad$ Maximum tillering & 64 & Shoot & 18 hills & 404 \\
Anthesis & 103 & Shoot & 5 hills & 781 \\
Milky ripening & 112 & Ear & 30 & 19 \\
End of ripening & 145 & Ear & 95 & 123 \\
in 1986 & & Ear & 948 & 2230 \\
3rd-leaf & 20 & Whole plant & 1100 plants & 310 \\
6th-leaf & 47 & Shoot & 1200 plants & 350 \\
Maximum tillering & 67 & Shoot & 13 hills & 330 \\
Panicle initiation & 88 & Shoot & 6 hills & 546 \\
Anthesis & 109 & Shoot & 5 hills & 1437 \\
Milky ripening & 117 & Ear & 200 & 178 \\
End of ripening & 166 & Ear & 300 & 435 \\
\hline
\end{tabular}

a One hill contained two to five plants.

until needed for analysis.

Extraction. Each sample was homogenized in methanol with a blender and extracted three times with methanol containing $1 \mathrm{mg} / 100 \mathrm{ml}$ of BHT. The filtered extract was combined and concentrated in vacuo, the concentrated solution being stored at $-20^{\circ} \mathrm{C}$ until analysis.

Purification for auxin analysis. The extracts from $100 \mathrm{~g}$ fr. wt. of shoots (6th-leaf stage) and ears (beginning of the milky ripening stage) collected in 1986 were concentrated. Water was added to the aqueous residue, and the solution was subjected to the solvent fractionation procedure shown in Fig. 1. The dichloromethane used in the procedure contained $1 \mathrm{mg} / 100 \mathrm{ml}$ of $\mathrm{BHT}$, and $2.5 \mathrm{M} \mathrm{HCl}$ was used for $\mathrm{pH}$ adjustment.

The concentrated acidic fraction was purified in three steps by HPLC. Each sample was dissolved in a small amount of tetrahydrofuran and injected into a column of Shodex GPC HF-2000P $+2001 \quad(8 \times 50 \mathrm{~mm}+20 \times 500$ $\mathrm{mm})$. The column was eluted with tetrahydrofuran at a flow rate of $3.5 \mathrm{ml} / \mathrm{min}$, and the eluate from $t_{R} 20.5$ to $24.5 \mathrm{~min}$ was collected.

The eluate was concentrated, dissolved in a small amount of $35 \%$ acetonitrile and injected into a column of Senshu Pak ODS-H-3151 $(8 \times 150 \mathrm{~mm})$. The column was eluted with a solvent of acetonitrile-acetic acid-water $(35: 0.5: 65)$ at a flow rate of $2 \mathrm{ml} / \mathrm{min}$, and four fractions corresponding to ICA $\left(t_{R} 4.8 \sim 6.0 \mathrm{~min}\right)$, IAA $\left(t_{R} 6.0 \sim\right.$ $7.5 \mathrm{~min})$, IPA and 4-chloro-IAA $\left(t_{R} 8.0 \sim 11.0 \mathrm{~min}\right)$, and IBA $\left(t_{R} 12.5 \sim 15.0 \mathrm{~min}\right)$ were collected. Each fraction was concentrated in vacuo to remove the acetonitrile and then

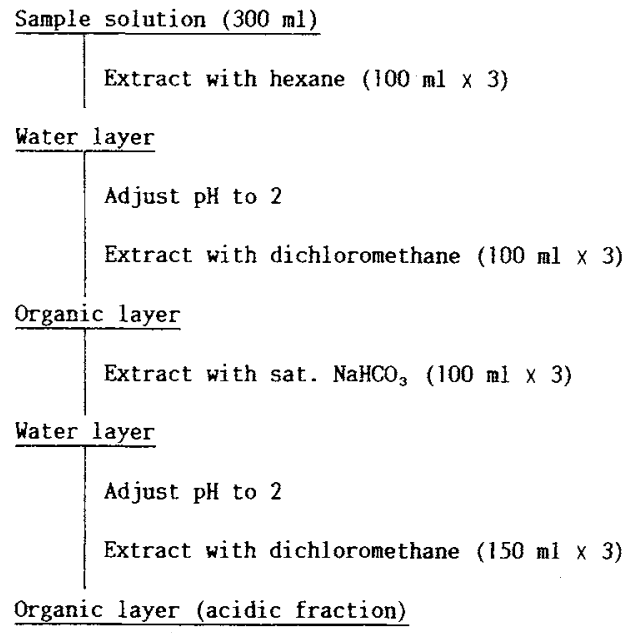

Fig. 1. Solvent Fractionation Procedure for Auxin Analysis.

Iyophilized.

The lyophilized sample was dissolved in a small amount of methanol and injected into a column of Nucleosil 5 $\mathrm{N}\left(\mathrm{CH}_{3}\right)_{2} \quad(6 \times 100 \mathrm{~mm})$. The column was eluted with methanol-acetic acid $(100: 0.2)$ at a flow rate of 1.5 $\mathrm{ml} / \mathrm{min}$, and the eluate was monitored with a fluorescence detector (excitation at $280 \mathrm{~nm}$ and emission at $360 \mathrm{~nm}$ ) or a UV detector for 4-chloro-IAA (monitored at $280 \mathrm{~nm}$ ). Authentic ICA, IBA, IPA, 4-chloro-IAA and IAA were eluted at the retention times of $3.4,5.9,7.6,9.9$ 
and $12.8 \mathrm{~min}$, respectively. Although the detection limit for IBA and IPA with the fluorescence detector was about $100 \mathrm{pg}$, these two auxins were not detected in the samples. Thus, the ICA, IAA and 4-chloro-IAA fractions were collected and subjected to GC-MS analysis.

$G C-M S$ analysis. A Hitachi M-80A mass spectrometer equipped with a Hitachi $003 \mathrm{~B}$ datalizer was used for $\mathrm{GC}$ MS analysis. A capillary column (chemically bonded OV$1,0.53 \mathrm{~mm} \times 15 \mathrm{~m}$ ) was employed with a carrier gas of helium at a flow rate of $30 \mathrm{ml} / \mathrm{min}$. Each sample was trimethylsilylated with $N$-methyl- $N$-trimethylsilyl-trifluoroacetamide (MSTFA, Pierce Chemical Company) and injected into the column. The column oven temperature was kept at $170^{\circ} \mathrm{C}$ until $1 \mathrm{~min}$ after the injection and then increased at a rate of $5^{\circ} \mathrm{C} / \mathrm{min}$. The retention times of authentic samples were $3.8 \mathrm{~min}$ for IAA-TMSi, $4.3 \mathrm{~min}$ for ICA-TMSi and $6.3 \mathrm{~min}$ for 4-chloro-IAA-TMSi.

Quantitative analysis of $I A A$. A methanol solution of the sample extract $(0.1 \sim 1 \mathrm{~g}$ fr. wt. equivalent) was concentrated in vacuo at room temperature, and 10 to $100 \mathrm{ng}$ of IPA or $18,000 \mathrm{dpm}$ of $\left[{ }^{3} \mathrm{H}\right] \mathrm{IAA}(30 \mathrm{Ci} / \mathrm{mmol}$, Amersham) was added as an internal standard. The aqueous residue of the sample was supplemented with $3 \mathrm{ml}$ of water saturated with $\mathrm{NaHCO}_{3}$, and extracted three times with $1 \mathrm{ml}$ of diethylether. The aqueous layer was then adjusted to $\mathrm{pH} 2$ with $2.5 \mathrm{M} \mathrm{HCl}$ and extracted with $1.5 \mathrm{ml}$ of diethylether to give a diethylether-soluble acidic fraction.

To analyze the IAA conjugate, the aqueous residue was supplemented with one-fourth volume of $5 \mathrm{~N} \mathrm{NaOH}$ and stirred for 1 hr at room temperature. After the hydrolysis, the solution was adjusted to $\mathrm{pH} 2$ with $2.5 \mathrm{M} \mathrm{HCl}$ and extracted with diethylether to give an acidic fraction in which the free IAA released from its conjugate was contained. The diethylether used in the procedure was distilled with $\mathrm{CaH}_{2}$ just before use and supplemented with $1 \mathrm{mg} / 10 \mathrm{ml}$ of BHT. A small amount of methanol was added to each acidic fraction, and the mixture was concentrated in vacuo at room temperature. The residue was dissolved in methanol and kept in a refrigerator until subjected to HPLC purification.

Samples containing IPA as an internal standard were subjected to purification with HPLC under the same conditions as those just described. The eluate from the Shodex column was collected from $t_{R} 21$ to $24 \mathrm{~min}$. The eluate from the Senshu Pak column was collected from $t_{R}$ 5.5 to $11 \mathrm{~min}$, concentrated, and analyzed by HPLC, using a column of Nucleosil $5 \mathrm{~N}\left(\mathrm{CH}_{3}\right)_{2}$ equipped with a fluorescence detector. The content of IAA was calculated from the ratio of the peak area of IAA to that of IPA.

Samples containing $\left[{ }^{3} \mathrm{H}\right] \mathrm{IAA}$ were purified by HPLC using a column of Nucleosil $5 \mathrm{~N}\left(\mathrm{CH}_{3}\right)_{2}$, each sample being dissolved in a small amount of methanol before injecting into the column. The column was eluted with a solvent system of methanol-acetic acid $(100: 0.2)$ at a flow rate of
$1.5 \mathrm{~min}$, and the eluate from $t_{R} 5.8$ to $7.8 \mathrm{~min}$ was collected. The fraction was concentrated under an $\mathrm{N}_{2}$ stream, dissolved in a small amount of acetonitrile-water $(35: 65)$ and injected into a column of Senshu Pak ODS-H-3151 equipped with a fluorescence detector. The column was eluted with a solvent system of acetonitrile-acetic acidwater $(35: 0.5: 65)$ at a flow rate of $1.5 \mathrm{ml} / \mathrm{min}$, and the amount of IAA was quantified by the peak area. The recovery of $\left[{ }^{3} \mathrm{H}\right]$ IAA was determined by measuring the radio activity in the collected IAA fraction ( $t_{R}$ $7.5 \sim 8.5 \mathrm{~min}$ ) using a liquid scintillation counter.

\section{Results and Discussion}

At the first stage of the investigation, IAA and several other auxins in the shoots and ears of rice (cultivar Nihonbare) were analyzed by HPLC and GC-MS. As shown in Figs. 2 and 3, IAA and ICA were identified in the ears of rice. They were also identified in the shoots, but at much lower levels than in the ears. From the analysis using HPLC and the fluorescence detector, the content of IAA was much higher than that of ICA. IPA, IBA and 4-chloro-IAA were not detected in any samples.

Bandurski et al. ${ }^{6)}$ have reported the occurrence of IAA in mature seeds of rice, and
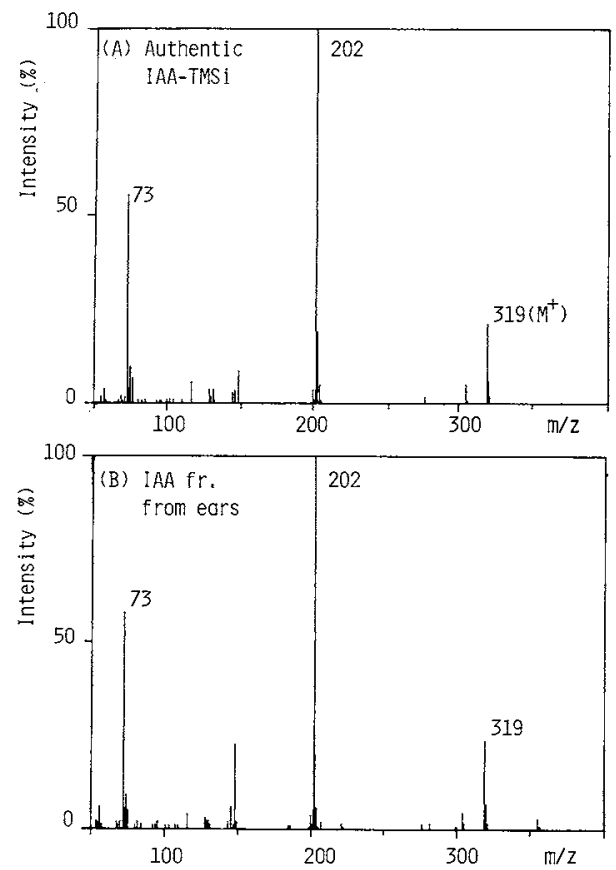

Fig. 2. Mass Spectrum of Authentic IAA-TMSi and Endogenous IAA in Ears of Rice. 

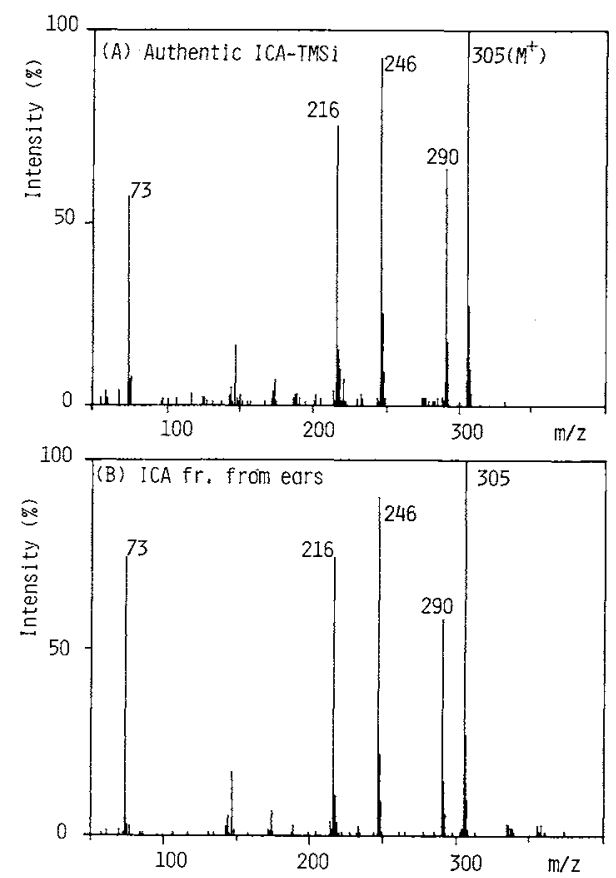

Fig. 3. Mass Spectrum of Authentic ICA-TMSi and Endogenous ICA in Ears of Rice.

Suzuki et $a l .{ }^{7)}$ have reported the identification of IAA and related compounds from rice bran. However, this is the first identification of IAA by GC-MS from a vegetative tissue of rice. The identification of ICA is also the first time in rice.

To study the fluctuation of IAA level, rice (cv. Nihonbare) was grown in a paddy field in 1985 and 1986, and its shoots and ears were collected at the several growth stages. Nakagawa $\mathrm{et} \mathrm{al}^{8)}$ have reported the procedure for analyzing IAA using HPLC and a fluorescence detector. By this method, $100 \mathrm{pg}$ of IAA can be detected, and IPA can be used as an internal standard. However, the large amount of impurities contained in shoots of rice would interfere with an exact quantification of IAA. Thus, we developed an improved purification procedure for the analysis of IAA in rice.

Each sample was supplemented with IPA as an internal standard and subjected to solvent fractionation to give an acidic fraction. Two steps of HPLC using columns of Shodex GPC HF-2001 and Senshu Pak ODS-H-3151 were
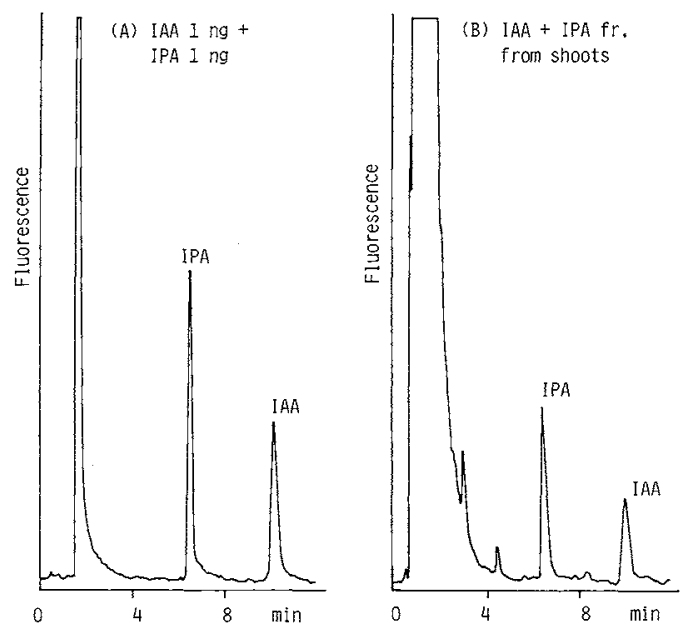

Fig. 4. HPLC Analysis of IAA in Rice.

Conditions of analysis were as follows: column, Nucleosil $5 \mathrm{~N}\left(\mathrm{CH}_{3}\right)_{2}(150 \times 6 \mathrm{~mm})$; solvent, $0.2 \%$ acetic acid in methanol; flow rate, $1.5 \mathrm{ml} / \mathrm{min}$; detection, fluorescence.

carried out to purify the acidic fraction, and IAA was quantified with HPLC using a column of Nucleosil $5 \mathrm{~N}\left(\mathrm{CH}_{3}\right)_{2}$ equipped with a fluorescence detector. As shown in Fig. 4, the IAA fraction was sufficiently purified with a high recovery (more than $40 \%$ and usually $60 \%$ of added IPA was recovered). The results of the analysis are shown in Fig. 5.

Although this analysis gave good recoveries of added IPA, it might have been possible for the recoveries of IAA and IPA throughout the analysis to be different. Thus, $\left[{ }^{3} \mathrm{H}\right] \mathrm{IAA}$ was added to the samples collected in 1986 and a reanalysis was run to confirm the results. After the solvent fractionation procedure, the acidic fraction was purified with HPLC using a column of Nucleosil $5 \mathrm{~N}\left(\mathrm{CH}_{3}\right)_{2}$, and IAA fraction was analyzed by HPLC using a column of Senshu Pak ODS-H-3151 equipped with a fluorescence detector. The usual recovery of $\left[{ }^{3} \mathrm{H}\right] \mathrm{IAA}$ was $60 \%$. The result of this reanalysis was almost consistent with the results shown in Fig. 5, the difference of IAA level determined by the two procedure being within $17 \%$ and showing that our results were certainly reliable.

Because Bandurski et al ${ }^{6)}$ reported the occurrence of esterified IAA in seeds of rice, the 

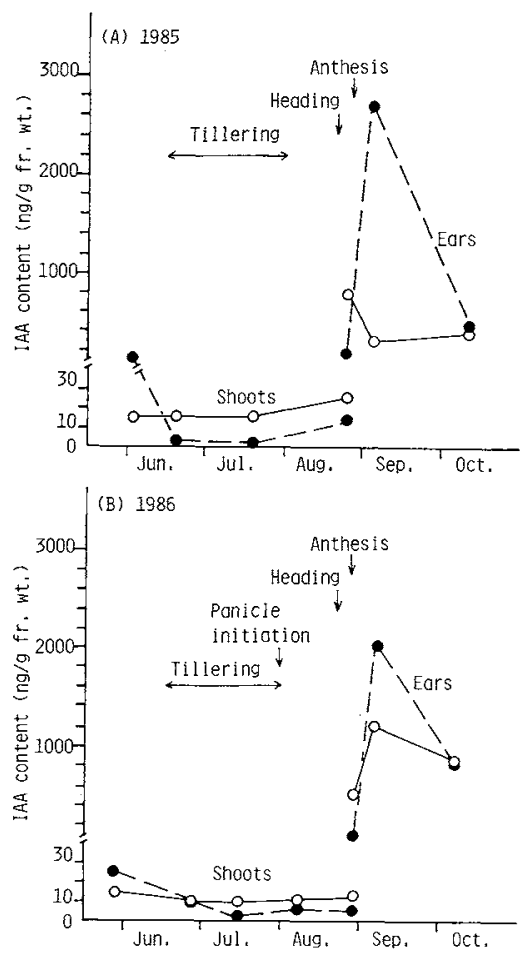

Fig. 5. Fluctuation Pattern of IAA and Its Conjugate Contents in Rice.

Open circles show the content of free IAA, and closed circles show the content of IAA conjugate.

aqueous residue of each sample obtained by the solvent fractionation procedure was subjected to hydrolysis with $1 \mathrm{~N} \mathrm{NaOH}$ for $1 \mathrm{hr}$ at room temperature. The hydrolyzed IAA was quantified using the foregoing method, producing the results shown in Fig. 5.

The level of IAA in shoots was $10 \sim 26 \mathrm{ng} / \mathrm{g}$ fr. wt. and did not show any significant difference in each year. The endogenous level of conjugated IAA in shoots was lower than that of free IAA, except at the 3rd-leaf stage.

In ears, the IAA content at the anthesis stage was $730 \mathrm{ng} / \mathrm{g}$ fr. wt. in 1985 and $540 \mathrm{ng} / \mathrm{g}$ fr. wt. in 1986. This increased to $1200 \mathrm{ng} / \mathrm{g}$ fr. wt. at the beginning of the milky ripening stage, and then decreased to $850 \mathrm{ng} / \mathrm{g}$ fr. wt. at the end of the ripening stage in 1986. However, no increase of IAA level at the milky ripening stage was observed in the 1985 samples. The level of conjugated IAA in ears rapidly increased during the week after the anthesis stage, and then decreased during seed maturation in both years' samples.

In this study, the IAA conjugate was quantified after hydrolysis, and its structure is still unknown. However, since Bandurski ${ }^{6)}$ reported that esterified IAA was hydrolyzed with $1 \mathrm{~N}$ $\mathrm{NaOH}$ and peptidyl IAA was not, the IAA conjugate detected in this study must have been an ester form.

From these results, the biosynthesis of IAA and its conjugate in ears is suggested to occur from the anthesis stage to the milky ripening stage, although the level of free IAA in ears at the milky ripening stage was much different between two years' samples. This discrepancy may have been due to a small difference in the stage of the samples, indicating that the rapid biosynthesis of IAA and the conversion of IAA to IAA conjugate must have occurred at this stage. It should be noted that the total contents of IAA and its conjugate in ears at this stage of the two years' samples were almost the same. (The total contents were $3040 \mathrm{ng} / \mathrm{g}$ fr. wt. in 1985 and $3400 \mathrm{ng} / \mathrm{g}$ fr. wt. in 1986.)

Bandurski et $a l .{ }^{6)}$ analyzed endogenous IAA in Zea mays and reported that the levels of IAA and its conjugate in immature and mature seeds were much higher than those in shoots, which is consistent with our results for rice. Bandurski ${ }^{9)}$ also reported studies on the biosynthesis, metabolism and transportation of IAA in seedlings of maize, and he speculated that IAA conjugate in the seeds regulated the growth of seedlings at the germination stage. In the case of rice, it is possible that IAA and its conjugate are stored in the seeds until germination, and then used for the early growth of seedlings. However, it is also possible that IAA regulates the development of embryo and endosperm because the level of IAA in ears was much higher than that in shoots, even at the anthesis stage. It is worthy of note that differentiation of the embryo and cell division of the endosperm occur during the first 10 days after anthesis. Moreover, the endogenous levels of cytokinins and abscisic acid were also high in developping seeds, ${ }^{2,5)}$ suggesting that 
the development of embryo and/or endosperm are regulated by a balance of the levels of these plant hormones. Further investigation will confirm the roles of plant hormones in the growth of rice.

Acknowledgments. We wish to thank Mr. Y. Esumi of The Institute of Physical and Chemical Research for his kind help in GC-MS analyses. We also thank Miss $Y$. Satake for her help in IAA analyses.

\section{References}

1) S. Kurogochi, N. Murofushi, Y. Ota and N. Takahashi, Planta, 146, 185 (1979).

2) Y. Suzuki, S. Kurogochi, N. Murofushi, Y. Ota and N. Takahashi, Plant Cell Physiol., 22, 1085 (1981).
3) M. Kobayashi, I. Yamaguchi, N. Murofushi, Y. Ota and N. Takahashi, Agric. Biol. Chem., 48, 2725 (1984).

4) M. Kobayashi, I. Yamaguchi, N. Murofushi, Y. Ota and N. Takahashi, Agric. Biol. Chem., 52, 1189 (1988).

5) M. Takagi, T. Yokota, N. Murofushi, Y. Ota and N. Takahashi, Agric. Biol. Chem., 49, 3271 (1985).

6) R. S. Bandurski and A. Schulze, Plant Physiol., 60, 211 (1977).

7) Y. Suzuki, H. Kinashi, S. Takeuchi and A. Kawarada, Phytochemistry, 16, 635 (1977).

8) S. Nakagawa, D. S. Tjokrokusumo, A. Sakurai, I. Yamaguchi, N. Takahashi and K. Shono, Plant Cell Physiol., 28, 485 (1987).

9) R. S. Bandurski, in "Plant Growth Substances 1979," ed. by F. Skoog, Springer-Verlag, Berlin, 1980, pp. $37 \sim 49$. 